\title{
Co-regulatory genes of CEBiP: a stress responsive gene for rice blast infection
}

\begin{abstract}
$\mathrm{CEBiP}$ is the major binding receptor for chitin elicitors and provides resistance against Magnaporthe oryzae, the causal agent of rice blast disease. Chitin elicitors lead to various defense responses in plants. CEBiP has been shown to interact with chitin elicitors and trigger the downstream defense responses. Here we identified genes that are co regulated with $\mathrm{CEBiP}$ and may play a role in defense mechanism for blast infection.

Microaray data were downloaded from NCBI GEO database and analyzed using R and bioconductor packages. $\mathrm{K}$ mean clustering was performed on the datasets and pearson correlation coefficients were calculated for genes falling in same cluster as CEBiP using SPSS. Genes identified for leaf tissues showed high correlation. For the dataset of blast infected root tissue, most of the identified genes in cluster were also correlated. The results suggest that defense mechanism to counteract chitin elicitor during blast infection is slightly different in leaf and roots, as the identified genes for both the tissues are different. Further, most of the identified genes have not been previously reported to be involved in rice defense and hence are novel targets for future studies.
\end{abstract}

Keywords: rice blast, microarray, co expression, stress response, chitin induced response
Volume 4 Issue 3 - 2016

\author{
Varshika Singh,' Afroz Alam,' Pramod \\ Katara, ${ }^{2}$ Vinay Sharma' \\ 'Department of Bioscience and Biotechnology, Banasthali \\ University, India \\ ${ }^{2}$ Center of Bioinformatics, University of Allahabad, India
}

\author{
Correspondence: Vinay Sharma, Department of Bioscience \\ and Biotechnology, Banasthali University, P.O. Banasthali \\ Vidyapith, Rajasthan 304 022, India, \\ Email vinaysharma30@yahoo.co.uk
}

Received: October 31, 2016 | Published: November 07, 2016

\section{Introduction}

Plants activate defense systems upon the recognition of microbeassociated molecular patterns (MAMPs). These are small, structurally conserved molecules within microbial species. Pattern recognition receptors (PRRs) located on the plasma membrane of plant cells, recognise the MAMPs. ${ }^{1-4}$ The PRRs are the receptor-like protein kinases (RLK) and receptor-like proteins (RLP) localized in the plasma membrane. ${ }^{3}$ Chitin is a constituent of fungal cell walls and acts as a carbohydrate MAMP, and elicits various defense responses in many plant species. ${ }^{5}$

In rice, CEBiP and OsCERK1 play key roles in the perception of chitin oligosaccharides ${ }^{6,7}$ LYP4 and LYP6 are also known to play role in the recognition of chitin in rice. ${ }^{8}$ LYP4 and LYP6 are also reported to bind peptidoglycan, and function as the receptors for both chitin and peptidoglycan. Knockdown of CEBiP, LYP4 or LYP6 expression have been shown to cause increase in the spread of the infection hyphae of the blast fungus. ${ }^{4,8}$ In the present study, we identified additional genes that may be involved along with CeBIP in defence response using microarray data.

\section{Materials and methodology}

\section{Dataset collection}

The microarray gene expression data from gene expression ominous (GEO) database at NCBI was downloaded. For comparison, we have choosen data from leaf and root tissues.

\section{Data analysis}

Microarray data downloaded were analysed with bioconductor packages using $\mathrm{R}$. The method used for affymetrix data (CEL files) normalization was Robust multi-array analysis (RMA) ${ }^{9}$ in the affy Bioconductor package. Functions for normalizing two-color agilent data are available in package limma; lowess (or loess) function was used. Further, k mean clustering was performed on whole datasets. Clusters having CeBIP in both the dataset were indentified and pearson correlation coefficient were calculated for genes of the same cluster using SPSS. The genes having correlation value $>0.75$ were selected as coexpressed.

Further analysis was done using blast2GO tool. mRNA Sequences downloaded from genbank were used as input and the coregulated genes were identified for the associated gene ontology terms. Also enzyme coding and kegg mapping options of the tool allowed to identify the already known pathways if any associated with the coregulated genes.

\section{Results}

\section{Identification of co-regulated genes}

It is assumed that the genes regulated similarly would have correlating expression patterns. We attempted to identify target genes for rice blast using microarray datasets. To investigate the correlations between genes in the datasets, pearson correlation analysis was used that gives the measure for how well the two genes correlate. The correlation tables for leaf and root data are shown in Table $1 \& 2$.

Three hypothetical genes (Os01g0566100, Os08g0242700 and Os10g0509100) were co regulated with CEBiP, along with Os05g0119300 (Glycine-rich protein A3), Os03g0222100 (MA3 domain-containing protein, putative, expressed) and Os07g0580500 (BZR1) in leaf tissue. The gene descriptions were downloaded from Oryzaexpress database (http://plantomics.mind.meiji.ac.jp/ OryzaExpress/). 
Table I Correlation coefficients for genes falling in same cluster as Cebip (Os03g0 I33400) in leaf tissue

The genes with correlation values higher than 0.75 are shown in bold

\begin{tabular}{|c|c|c|c|c|c|c|c|}
\hline $\begin{array}{l}\text { 7:OS 07G } \\
0580500\end{array}$ & 0.977 & 0.965 & 0.964 & 0.979 & 0.976 & 0.961 & I \\
\hline $\begin{array}{l}\text { 6:OSIOG } \\
0509100\end{array}$ & 0.985 & 0.969 & 0.99 & 0.991 & 0.963 & I & 0.961 \\
\hline $\begin{array}{l}\text { 5:OS08G } \\
0242700\end{array}$ & 0.985 & 0.988 & 0.976 & 0.973 & I & 0.963 & 0.976 \\
\hline $\begin{array}{l}\text { 4:OS03G } \\
0222100\end{array}$ & 0.989 & 0.97 & 0.991 & 1 & 0.973 & 0.991 & 0.979 \\
\hline $\begin{array}{l}\text { 3:OS03G } \\
0133400\end{array}$ & 0.989 & 0.985 & 1 & 0.991 & 0.976 & 0.99 & 0.964 \\
\hline $\begin{array}{l}2: \text { OS05G } \\
119300\end{array}$ & 0.986 & 1 & 0.985 & 0.97 & 0.988 & 0.969 & 0.965 \\
\hline \multirow[t]{3}{*}{$\begin{array}{l}\text { I:OSOIG } \\
0566100\end{array}$} & 1 & 0.986 & 0.989 & 0.989 & 0.985 & 0.985 & 0.977 \\
\hline & I:OSOIG & 2:OS05G & 3:OS03G & 4:OS03G & 5:OS08G & 6:OSIOG & 7:OS07G \\
\hline & 0566100 & 0119300 & 0133400 & 0222100 & 0242700 & 0509100 & 0580500 \\
\hline
\end{tabular}

Following datasets were retrieved from GEO for analysis.

\begin{tabular}{lll}
\hline S. no. & GSE ID & Description \\
\hline I & GSE85 I8 & Rice leaf sheath at 36 hrs after inoculation rice with blast fungus M. oryzae using agilent array technologies. ${ }^{12}$ \\
2 & GSE I836I & Infected (with M. oryzae) and non infected roots of rice at 2, 4, and 6 days post-inoculation using affymetrix technology. ${ }^{13}$
\end{tabular}

Table 2 Correlation coefficients for genes falling in same cluster as Cebip (Os03g0I33400) in root tissue. The genes with correlation values higher than 0.75 are shown in bold

\begin{tabular}{|c|c|c|c|c|c|c|c|c|c|c|c|c|c|c|}
\hline 14:Os04g0674000 & 0.753 & 0.947 & 0.873 & 0.885 & 0.775 & 0.933 & 0.812 & 0.923 & 0.917 & 0.95 & 0.897 & 0.831 & 0.923 & I \\
\hline |3:Os07g0557| 00 & 0.837 & 0.872 & 0.874 & 0.894 & 0.716 & 0.882 & 0.782 & 0.9 & 0.951 & 0.946 & 0.862 & 0.829 & I & 0.923 \\
\hline I2:Os03g0757900 & 0.865 & 0.855 & 0.796 & 0.83 & 0.849 & 0.913 & 0.843 & 0.926 & 0.908 & 0.769 & 0.966 & 1 & 0.829 & 0.831 \\
\hline II:Os07g058 I 000 & 0.82 & 0.89 & 0.81 & 0.82 & 0.8 & 0.95 & 0.87 & 0.9 & 0.92 & 0.85 & I & 0.97 & 0.86 & 0.9 \\
\hline I0:Os0 I g0292200 & 0.72 & 0.83 & 0.88 & 0.85 & 0.61 & 0.84 & 0.7 & 0.86 & 0.88 & I & 0.85 & 0.77 & 0.95 & 0.95 \\
\hline $\begin{array}{l}\text { 9:Os0Ig } \\
0830700\end{array}$ & 0.86 & 0.93 & & 0.93 & 0.87 & 0.95 & 0.91 & 0.95 & I & 0.88 & 0.92 & 0.91 & 0.95 & 0.92 \\
\hline $\begin{array}{l}\text { 8:Os0lg } \\
0290700\end{array}$ & 0.83 & 0.92 & 0.81 & 0.93 & 0.87 & 0.95 & 0.91 & 0.95 & I & 0.88 & 0.92 & 0.91 & 0.95 & 0.92 \\
\hline 7:Os06g066 & 0.75 & 0.89 & 0.63 & 0.8 & 0.91 & 0.92 & 1 & 0.85 & 0.91 & 0.7 & 0.87 & 0.84 & 0.78 & 0.81 \\
\hline $\begin{array}{l}\text { 6:Os02g } \\
072\end{array}$ & 0.8 & 0.96 & 0.77 & 0.86 & 0.88 & I & 0.92 & 0.92 & 0.95 & 0.84 & 0.95 & 0.91 & 0.88 & 0.93 \\
\hline $\begin{array}{l}\text { 5:Os03g } \\
0133400\end{array}$ & 0.82 & 0.9 & 0.67 & 0.8 & 1 & 0.88 & 0.91 & 0.88 & 0.87 & 0.61 & 0.8 & 0.85 & 0.72 & 0.78 \\
\hline 4:Os03g072 & 0.83 & 0.9 & 0.81 & I & 0.8 & 0.86 & 0.8 & 0.93 & 0.93 & 0.85 & 0.82 & 0.83 & 0.89 & 0.89 \\
\hline $\begin{array}{l}\text { 3:Os02g07 } \\
66700\end{array}$ & $0.87 I$ & 0.81 & I & 0.811 & 0.669 & 0.767 & 0.628 & 0.847 & 0.812 & 0.875 & 0.81 & 0.796 & 0.874 & 0.873 \\
\hline $\begin{array}{l}\text { 2:Os07g0I } \\
76200\end{array}$ & 0.817 & I & 0.81 & 0.896 & 0.9 & 0.964 & 0.893 & 0.916 & 0.929 & 0.828 & 0.885 & 0.855 & 0.872 & 0.947 \\
\hline \multirow[t]{2}{*}{ I:Os04g0244800 } & I & 0.817 & $0.87 I$ & 0.826 & 0.82 & 0.795 & 0.748 & 0.831 & 0.861 & 0.719 & 0.815 & 0.865 & 0.837 & 0.753 \\
\hline & $\begin{array}{l}\text { I:Os04g } \\
244800\end{array}$ & $\begin{array}{r}02: \text { Os078 } \\
0176200\end{array}$ & $\begin{array}{l}3: \text { Os02g } \\
00766700\end{array}$ & $\begin{array}{l}4: \text { Os03g } \\
072\end{array}$ & $\begin{array}{r}5: O s 03 \\
013340\end{array}$ & $\begin{array}{l}\text { g 6:Os02\& } \\
0072\end{array}$ & $7: 0 s 06.66$ & $\begin{array}{l}8: \text { Os0 Ig } \\
290700\end{array}$ & $\begin{array}{c}09: \text { Os0 Ig0 } \\
830700\end{array}$ & $\begin{array}{l}10: O s 01 \mathrm{~g} \\
0292200\end{array}$ & $\begin{array}{l}1 \mathrm{I}: \text { Os07g } \\
0581000\end{array}$ & $\begin{array}{c}\text { I I2:Os03g } \\
0757900\end{array}$ & $\begin{array}{l}\text { g I3:Os07 } \\
0557100\end{array}$ & $\begin{array}{l}g \text { I } 4: \text { Os04g } \\
0674000\end{array}$ \\
\hline
\end{tabular}

The co-expressed genes in root tissue were Os04g0244800 (heavy Os03g0726500 (protein of unknown function DUF81 domain metal transport/detoxification protein domain containing protein), containing protein), Os06g0661900 (protein of unknown function Os07g0176200 (tesmin/TSO1-like, CXC domain containing protein, DUF266), Os01g0290700 (similar to CjMDR1), Os01g0830700 
(protein of unknown function DUF231), Os07g0581000 (protein of unknown function DUF250), Os03g0757900 (similar to UDP-glucose 6-dehydrogenase), Os04g0674000 (similar to H0403D02.10 protein).

\section{Gene Ontology analysis and pathway analysis}

Blast2GO was used for analysis and the data are compiled in Table
$3 \& 4$ for leaf and root tissue respectively. The results for BLAST analysis are against the rice protein database and then the GO (gene ontology) terms database. For leaf tissue data no enzyme codes were identified for KEGG pathways. Thus none of these genes can be predicted to be involved in any of the known KEGG pathways. CeBiP too did not have an enzyme code associated with it.

Table 3 Blast2GO results showing values for BLAST similarity search, GO terms, enzyme code analysis and KEGG pathway analysis for leaf tissue data

\begin{tabular}{|c|c|c|c|c|c|c|c|c|}
\hline Description & $\begin{array}{l}\text { Seq. } \\
\text { length }\end{array}$ & \#Hits & $\begin{array}{l}\text { Min. } \\
\text { eValue }\end{array}$ & $\begin{array}{l}\text { Mean } \\
\text { similarity }\end{array}$ & \#GOs & GOs & $\begin{array}{l}\text { Enzyme } \\
\text { codes }\end{array}$ & $\begin{array}{l}\text { KEGG } \\
\text { pathway }\end{array}$ \\
\hline Osl2g0I52700 & 1779 & 1 & $\begin{array}{l}\text { I.3IE- } \\
35\end{array}$ & $72.00 \%$ & 2 & P, metabolic process; C, chloroplast part & - & - \\
\hline Oslog0509100 & || $2 \mid$ & 1 & $\begin{array}{l}1.18 \mathrm{E}- \\
113\end{array}$ & $100.00 \%$ & 0 & - & - & - \\
\hline Os06g0552300 & 1206 & 3 & $\begin{array}{l}\text { I.29E- } \\
34\end{array}$ & $54.33 \%$ & 4 & $\begin{array}{l}\mathrm{P} \text {, carbohydrate metabolic process; } \mathrm{F} \text {, catalytic } \\
\text { activity; } \mathrm{P} \text {, polysaccharide catabolic process; } \mathrm{F} \text {, } \\
\text { beta-amylase activity }\end{array}$ & - & - \\
\hline Os08g0242700 & 926 & 0 & & & 0 & - & - & - \\
\hline Os03g0222100 & 2587 & 5 & 0 & $67.00 \%$ & I & P:RNA metabolic process & - & - \\
\hline Os03g0I 33400 & $|5| \mid$ & 7 & 0 & $55.43 \%$ & 7 & $\begin{array}{l}\text { P, defense response; } \mathrm{P} \text {, cell wall macromolecule } \\
\text { catabolic process; } \mathrm{C} \text {, integral component } \\
\text { of membrane; } \mathrm{F} \text {, chitin binding; F, protein } \\
\text { self-association; C, cytoplasmic, membrane- } \\
\text { bounded vesicle; C, plasma membrane }\end{array}$ & - & - \\
\hline Os0lg0566100 & $|68|$ & I & 0 & $100.00 \%$ & 0 & - & - & - \\
\hline
\end{tabular}

$\mathrm{P}$ represents associated biological processes, $\mathrm{F}$ represent molecular processes and $\mathrm{C}$ represent cellular components in $\mathrm{GO}$ description.

Table 4 Blast2GO results showing values for BLAST similarity search, GO terms, enzyme code analysis and KEGG pathway analysis for root tissue data.

\begin{tabular}{|c|c|c|c|c|c|c|c|c|}
\hline Seq. description & $\begin{array}{l}\text { Seq. } \\
\text { length }\end{array}$ & \#Hits & $\begin{array}{l}\text { Min. } \\
\text { eValue }\end{array}$ & $\begin{array}{l}\text { Mean } \\
\text { similarity }\end{array}$ & \#GOs & GOs & $\begin{array}{l}\text { Enzyme } \\
\text { codes }\end{array}$ & KEGG pathway \\
\hline Os07g058I000 & 1710 & 16 & 0 & $58.75 \%$ & 4 & $\begin{array}{l}\mathrm{P} \text {, response to nematode; } \mathrm{C} \text {, } \\
\text { intracellular membrane-bounded } \\
\text { organelle; } \mathrm{C} \text {, cytoplasmic part; } \mathrm{C} \text {, } \\
\text { integral to membrane }\end{array}$ & - & \\
\hline Os07g0557I00 & 1681 & 3 & 0 & $86.33 \%$ & 3 & $\begin{array}{l}\text { P, transport; C, integral to } \\
\text { membrane; F, nucleoside } \\
\text { transmembrane transporter } \\
\text { activity }\end{array}$ & - & \\
\hline Os07g0I76200 & 2760 & 6 & 0 & $64.00 \%$ & I & C, plastid & & \\
\hline Os06g0661900, partial & 2021 & 14 & 0 & $62.50 \%$ & 3 & $\begin{array}{l}\text { C, membrane; F, } \\
\text { acetylglucosaminyltransferase } \\
\text { activity; C, mitochondrion }\end{array}$ & EC:2.4.I & \\
\hline Os04g0674000, partial & 1145 & I & $\begin{array}{l}5.01 \mathrm{E}- \\
92\end{array}$ & $100.00 \%$ & I & C, plastid & - & \\
\hline Os04g0244800 & 801 & 20 & $\begin{array}{l}3.03 \mathrm{E}- \\
110\end{array}$ & $65.90 \%$ & 3 & $\begin{array}{l}\mathrm{F} \text {, metal ion binding; } \mathrm{P} \text {, metal ion } \\
\text { transport; } \mathrm{C} \text {, plasma membrane }\end{array}$ & - & \\
\hline Os03g0757900 & 1942 & 3 & 0 & $98.67 \%$ & 8 & $\begin{array}{l}\text { F, UDP-glucose 6-dehydrogenase } \\
\text { activity; C, cytosol; F, NAD } \\
\text { binding; C, cell wall; P, } \\
\text { oxidation-reduction process; C, } \\
\text { cytoplasmic membrane-bounded } \\
\text { vesicle; P, UDP-glucuronate } \\
\text { biosynthetic process; C, nucleus }\end{array}$ & EC:1.1.1.22 & $\begin{array}{l}\text { Amino sugar and } \\
\text { nucleotide sugar } \\
\text { metabolism } \\
\text { Ascorbate } \\
\text { and aldarate } \\
\text { metabolism } \\
\text { Pentose and } \\
\text { glucuronate } \\
\text { interconversions }\end{array}$ \\
\hline Os03g0726500 & 2019 & 5 & 0 & $70.60 \%$ & 3 & $\begin{array}{l}\mathrm{C} \text {, cytoplasmic membrane- } \\
\text { bounded vesicle; } \mathrm{C} \text {, integral to } \\
\text { membrane; } \mathrm{C} \text {, mitochondrion }\end{array}$ & - & \\
\hline
\end{tabular}


Table Continued....

\begin{tabular}{|c|c|c|c|c|c|c|c|c|}
\hline Seq. description & $\begin{array}{l}\text { Seq. } \\
\text { length }\end{array}$ & \#Hits & $\begin{array}{l}\text { Min. } \\
\text { eValue }\end{array}$ & $\begin{array}{l}\text { Mean } \\
\text { similarity }\end{array}$ & \#GOs & GOs & $\begin{array}{l}\text { Enzyme } \\
\text { codes }\end{array}$ & KEGG pathway \\
\hline & & & & & & $\begin{array}{l}\mathrm{P} \text {, defense response; } \mathrm{P} \text {, cell } \\
\text { wall macromolecule catabolic } \\
\text { process; } \mathrm{C} \text {, integral to }\end{array}$ & & \\
\hline Os03g0I33400 & $|5| \mid$ & 7 & 0 & $55.43 \%$ & 7 & $\begin{array}{l}\text { membrane; F, chitin binding; } \\
\text { F, protein self-association; C, } \\
\text { cytoplasmic membrane-bounded } \\
\text { vesicle; C, plasma membrane }\end{array}$ & - & \\
\hline Os02g0766700 & 1827 & 9 & $\begin{array}{l}4.02 E- \\
15 I\end{array}$ & $62.33 \%$ & 7 & $\begin{array}{l}\text { F, transcription regulatory region } \\
\text { DNA binding; P, response to } \\
\text { abiotic stimulus; } P \text {, regulation of } \\
\text { transcription, DNA-dependent; F, } \\
\text { sequence-specific DNA binding; } \\
\text { F, sequence-specific DNA binding } \\
\text { transcription factor activity; } P \text {, } \\
\text { abscisic acid mediated signaling } \\
\text { pathway; } P \text {, response to stress }\end{array}$ & - & \\
\hline Os02g072I 700 & 1012 & 5 & $\begin{array}{l}9.49 \mathrm{E}- \\
91\end{array}$ & $72.80 \%$ & 6 & $\begin{array}{l}\text { P, pollen tube reception; } \mathrm{P} \text {, pollen } \\
\text { tube guidance; } \mathrm{C} \text {, cytoplasmic } \\
\text { membrane-bounded vesicle; } \mathrm{C} \text {, } \\
\text { plasma membrane; } \mathrm{P} \text {, synergid } \\
\text { death; } \mathrm{P} \text {, double fertilization } \\
\text { forming a zygote and endosperm }\end{array}$ & - & \\
\hline & & & & & & $\begin{array}{l}\text { C, mitochondrion; } \mathrm{P} \text {, cellular } \\
\text { macromolecule metabolic } \\
\text { process; C, plastid; P, cell wall }\end{array}$ & & \\
\hline Os0Ig0830700 & 2019 & 20 & 0 & $63.20 \%$ & 6 & $\begin{array}{l}\text { organization or biogenesis; } \\
\mathrm{P} \text {, response to freezing; } \mathrm{P} \\
\text { polysaccharide metabolic } \\
\text { process }\end{array}$ & - & \\
\hline & & & & & & $\begin{array}{l}\mathrm{P} \text {, response to salt stress; } \mathrm{F} \\
\text { protein tyrosine kinase activity; } \\
\mathrm{P} \text {, protein phosphorylation; } \mathrm{F} \\
\text { protein serine/threonine kinase } \\
\text { activity; } \mathrm{P} \text {, signal transduction; }\end{array}$ & & \\
\hline Os0Ig0292200 & 1785 & 20 & 0 & $67.25 \%$ & II & $\begin{array}{l}\text { P, response to inorganic } \\
\text { substance; } \mathrm{P} \text {, response to } \\
\text { extracellular stimulus; } \mathrm{P}, \\
\text { response to abscisic acid } \\
\text { stimulus; C, plasma membrane; F, } \\
\text { ATP binding; C, mitochondrion }\end{array}$ & $\begin{array}{l}\text { EC:2.7.10; } \\
\text { EC:2.7.II }\end{array}$ & \\
\hline Os0 Ig0290700, partial & 730 & 20 & $\begin{array}{l}4.62 \mathrm{E}- \\
93\end{array}$ & $77.95 \%$ & 23 & $\begin{array}{l}\text { P, root hair elongation; C, } \\
\text { vacuolar membrane; P,ATP } \\
\text { catabolic process; P, response to } \\
\text { cytokinin stimulus; C, chloroplast } \\
\text { envelope; P, response to } \\
\text { aluminum ion; P, auxin efflux; } \\
\text { C, cytoplasmic membrane- } \\
\text { bounded vesicle; C, plasma } \\
\text { membrane; P, gravitropism; } \\
\text { C, integral to membrane; F, } \\
\text { auxin efflux transmembrane } \\
\text { transporter activity; C, } \\
\text { mitochondrion; C,ATP-binding } \\
\text { cassette (ABC) transporter } \\
\text { complex; } \\
\text { P, acropetal auxin transport; } \\
\text { F,ATP binding; P, basipetal } \\
\text { auxin transport; F, xenobiotic- } \\
\text { transporting ATPase activity; } P \text {, } \\
\text { response to stress; C, plant-type } \\
\text { vacuole; } \\
\text { P, cellular metal ion homeostasis; } \\
\text { P, transmembrane transport; } \\
\text { F, oligopeptide-transporting } \\
\text { ATPase activity }\end{array}$ & $\begin{array}{l}\text { EC:3.6.3.44; } \\
\text { EC:3.6.3.23 }\end{array}$ & \\
\hline
\end{tabular}

P represents associated biological processes, $\mathrm{F}$ represent molecular processes and $\mathrm{C}$ represent cellular components in GO description.

Citation: Singh V,Alam A, Katara P, et al. Co-regulatory genes of CEBiP: a stress responsive gene for rice blast infection. MOJ Proteomics Bioinform. 
For root tissues few of the coregulated genes were identified with enzyme codes and also Kegg Pathways associated with one of them have been provided by Blast2GO tool. Os03g0757900 (similar to UDP-glucose 6-dehydrogenase) was identified with KEGG pathways; amino sugar and nucleotide sugar metabolism, ascorbate and aldarate metabolism, pentose and glucuronate interconversions. Thus we can predict that the identified coregulated genes must also share common functions associated with these pathways.

\section{Discussion}

Chitin is a well-known inducer of immune responses in plants and significant advances in knowledge of the molecular mechanisms of chitin perception and chitin-triggered immunity in plants have been achieved..$^{10}$ However, these processes are still not completely known. The different genes involved along with the signaling components remain largely unknown.

CeBIP, OsCERK1, OsRAC1, OsLYP4 and OsLYP6, have been previously reported to be involved in the defence mechanism but this knowledge is limited. ${ }^{11}$ Using coexpression analysis we established a direct link between the genes that must be coregulated and can be further analysed and verified using wet lab techniques. According to our analysis a defined pathway for chitin induced defence response is still not available and hence need extensive work to be done in this regard. Also all the genes identified in our study provides a strong starting point for the same.

\section{Conclusion}

Microarray analysis is a high throughput technique that leads to the generation of a large amount of data and can be used by other researchers for further analysis. Here we downloaded freely available data from NCBI GEO database and identified coexpressed genes of CeBIP in rice and root tissues. The genes identified were different for the two datasets. Our study provides an insight into the chitin induced defence mechanism. Further the genes identified are novel targtes for further studies as they have not been identified in chitin induced defence mechanism previously.

\section{Acknowledgements}

Authors gratefully acknowledge the liberal use of bioinformatics facilities available at DBT, Government of India, and Center for Bioinformatics at Department of Bioscience and Biotechnology, Banasthali University, India during the completion of this research work.

\section{Conflict of interest}

The author declares no conflict of interest.

\section{References}

1. Boller T, He SY. Innate Immunity in Plants: An Arms Race between Pattern Recognition Receptors in Plants and Effectors in Microbial Pathogens. Science. 2009;324(5928):742-744.

2. Tena G, Boudsocq M, Sheen J. Protein kinase signaling networks in plant innate immunity. Curr Opin Plant Biol. 2011;14(5):51.

3. Monaghan J, Zipfel C. Plant pattern recognition receptor complexes at the plasma membrane. Curr Opin Plant Biol. 2012;15(4):349-357.

4. Kishimoto K, Kouzai Y, Kaku H, et al. Perception of the chitin oligosaccharides contributes to disease resistance to blast fungus Magnaporthe oryzae in rice. Plant J. 2010;64(2):343-354.

5. Kombrink A, Sánchez-Vallet A, Thomma BP. The role of chitin detection in plant-pathogen interactions. Microbes Infect. 2011;13(14-15):11681176.

6. Kaku H, Nishizawa Y, Ishii-Minami N, et al. Plant cells recognize chitin fragments for defense signaling through a plasma membrane receptor. Proc Natl Acad Sci U S A. 2006;103(29):11086-11091.

7. Shimizu T, Nakano T, Takamizawa D, et al. Two LysM receptor molecules, CEBiP and OsCERK1, cooperatively regulate chitin elicitor signaling in rice. Plant J. 2010;64(2):204-214.

8. Liu B, Li JF, Ao Y, et al. Lysin motif-containing proteins LYP4 and LYP6 play dual roles in peptidoglycan and chitin perception in rice innate immunity. Plant Cell. 2012;24(8):3406-3419.

9. Irizarry RA, Hobbs B, Collin F, et al. Exploration, normalization, and summaries of high density oligonucleotide array probe level data. Biostatistics. 2003;4(2):249-264.

10. Sánchez-Vallet A, Mesters JR, Thomma BP. The battle for chitin recognition in plant-microbe interactions. FEMS Microbiol Rev. 2015;39(2):171-183.

11. Kouzai Y, Kaku H, Shibuya N, et al. Expression of the chimeric receptor between the chitin elicitor receptor $\mathrm{CEBiP}$ and the receptor-like protein kinase $\mathrm{Pi}-\mathrm{d} 2$ leads to enhanced responses to the chitin elicitor and disease resistance against Magnaporthe oryzae in rice. Plant Mol Biol. 2013;81(3):287-295.

12. Marcel S, Sawers R, Oakeley E, et al. Tissue-adapted invasion strategies of the Rice blast fungus Magnaporthe oryzae. Plant Cell. 2010;22(9):3177-3187.

13. Mosquera $\mathrm{G}$, Giraldo $\mathrm{MC}$, Khang $\mathrm{CH}$, et al. Interaction transcriptome analysis identifies Magnaporthe oryzae BAS1-4 as biotrophy-associated secreted proteins in rice blast disease. Plant Cell. 2009;21(4):1273-1290. 